\title{
Associations of dietary habits, physical activity and cognitive views with gestational diabetes mellitus among Chinese women
}

\author{
Qing $\mathrm{Li}^{1,2,}+$, Ribo Xiong ${ }^{2}+$, Liang Wang ${ }^{3}$, Junying $\mathrm{Cui}^{2}$, Linna Shi ${ }^{1, *}$, Yungang Liu ${ }^{2, *}$ \\ and Bingde Luo ${ }^{2, *}$ \\ 'Department of Dietetics, Nanfang Hospital, Southern Medical University, 1838 N. Guangzhou DaDao, \\ Guangzhou 510515, People's Republic of China: ${ }^{2}$ School of Public Health and Tropical Medicine, Southern \\ Medical University, 1023 S. Shatai Road, Guangzhou 510515, People's Republic of China: ${ }^{3}$ Department of \\ Biostatistics and Epidemiology, College of Public Health, East Tennessee State University, Johnson City, TN, USA
}

Submitted 30 January 2013: Final revision received 2 June 2013: Accepted 12 June 2013: First published online 7 August 2013

\begin{abstract}
Objective: To evaluate the relationship between dietary habits, physical activity and cognitive views and the risk of gestational diabetes mellitus (GDM) in Chinese women.

Design: A cross-sectional study to explore the potential risk factors of GMD through the International Physical Activity Questionnaire, an FFQ and a selfdesigned structured questionnaire, respectively.

Setting: Guangzhou, Guangdong Province, China.

Subjects: Chinese pregnant women ( $n$ 571) who underwent a 75-g oral glucose tolerance test at their 24 th to 28th gestational week.

Results: Thirteen per cent of the investigated women were identified as having GDM, and an increased intake of local featured foods and lower physical activity were observed in the GDM-positive group $v$. the GDM-negative group. Women who regarded early-pregnancy morning sickness as relevant to fetal abnormalities and those with unlimited dietary intake after the ending of morning sickness both had an increased risk for $\operatorname{GDM}(P=0 \cdot 018$ and $P=0 \cdot 038$, respectively). After multiple logistic regression analysis, cognitive views for unlimited food intake subsequent to morning sickness, increased consumption of energy-dense snack foods and highglycaemic-index fruits were strongly associated with the risk of GDM (OR $=1.911$, $P=0 \cdot 032 ; \mathrm{OR}=1 \cdot 050, P=0 \cdot 001$; and $\mathrm{OR}=1 \cdot 002, P=0 \cdot 017$, respectively).

Conclusions: Local featured foods and incorrect cognitive views on pregnancyrelated health were closely related to the risk of GDM in Chinese women. Intensive health education about pregnancy physiology and reasonable dietary and physical exercise behaviours should be strengthened for the control of GDM.
\end{abstract}

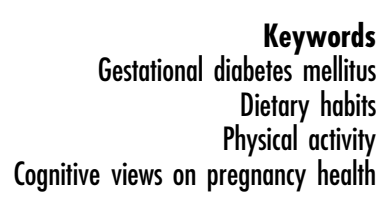

Gestational diabetes mellitus (GDM) is glucose intolerance of any degree appearing for the first time during pregnancy ${ }^{(1)}$. It is the most common complication during pregnancy, affecting approximately $2-12 \%$ of pregnant women $^{(2-4)}$. One in ten pregnancies worldwide is associated with diabetes, of which $90 \%$ are diagnosed as GDM. Approximately $30 \%$ of pregnancies in high-risk individuals, including advanced maternal age, overweight or obese mothers, and those with a positive family history of diabetes, may develop diabetes ${ }^{(5-7)}$. Women with GDM have an increased risk of subsequent development of type 2 diabetes and other maternal complications ${ }^{(8-10)}$. Similarly, offspring of mothers with GDM are at greater risk of fetal/neonatal morbidity and consecutive obesity and glucose intolerance ${ }^{(11,12)}$. Overall, the prevalence of GDM

$\dagger$ These two authors contributed equally to this work. has been rising everywhere, with a dramatic increase seen in mainland China in the past 30 years ${ }^{(13-15)}$.

The duration of pregnancy is accompanied with complex physiological, social and emotional changes. About $80 \%$ of pregnant women suffer from some degree of morning sickness, nausea and vomiting in the first trimester, thereby resulting in reduced maternal food intake ${ }^{(16,17)}$. Chinese pregnant women commonly consider that this phenomenon may result in fetal developmental retardation, low birth weight and stillbirth ${ }^{(18,19)}$. Based on this cognition, they usually try their best to increase dietary intake as soon as their morning sickness gets resolved at the beginning of the second trimester. To our knowledge, such diet modification, the related cognitive views and their associations with increased risk of GDM in Chinese women have not been reported.

With the socio-economic improvement in China in the past decade, people have experienced rapid lifestyle 
changes leading to a sedentary lifestyle and a Western-like dietary pattern ${ }^{(8)}$. Consequently, the number of women with increased body weight, obesity and diabetes (including GDM) has risen tremendously over the past 30 years ${ }^{(20,21)}$. It has been reported that some behavioural factors, such as dietary intake, low physical activity, weight gain and substance use, are associated with the risk of GDM among pregnant women ${ }^{(22-24)}$. However, most of the studies in this respect were carried out on Caucasian women $^{(25-28)}$ and only a few studies were conducted on Asian populations, especially in relation to Chinese society. In addition, studies to understand the association of cognitive views on pregnancy health with the incidence of GDM are extremely lacking. The main contents of the current family planning policy in China are advocating delayed marriage ( $>23$ years) and delayed childbearing ( $>24$ years). Additionally, Chinese young women are expected by their parents and society to finish all their planned education (including $\mathrm{PhD}$ studies in some cases) before they get married; and young couples are expected to have their own apartments before planning to have a baby. Therefore, the average age for Chinese women to have babies is relatively advanced (e.g. 30.63 years in a recent report $)^{(29)}$. It appears that Chinese pregnant women are probably more predisposed to GDM. Moreover, only a short time (usually less than $10 \mathrm{~min}$ ) is allocated for prenatal care to pregnant women during their first trimester. This results in minimal physician-patient interaction and subsequent few case-specific health educations for Chinese pregnant women. In the current study we hypothesized that there is a significant diet modification among Chinese pregnant women during their second trimester as a compensatory phenomenon to combat the insufficient food intake caused by morning sickness during the first trimester. Moreover, the associations of dietary habits, physical activity and cognitive views on health during pregnancy in relation to the increased risk of GDM were assessed locally in the region of Guangzhou, Guangdong Province, China.

\section{Methods}

\section{Study design and participants}

The study was a cross-sectional one involving 571 pregnant women who underwent a 2-h, 75-g oral glucose tolerance test (OGTT). Pregnant women were recruited from the out-patient service of the Department of Gynecology and Obstetrics and the Department of Endocrinology at Nanfang Hospital. Women with the following conditions were included: aged between 18 and 40 years old, pregnancy at gestation between 24 weeks and 28 weeks, and fully interviewed. The following cases were excluded from the study: having been diagnosed with type 2 diabetes and current treatment with medications supposed to adversely influence glucose tolerance (e.g. prednisone or other steroids). GDM was diagnosed according to the American Diabetes Association's new recommendations for the 2-h, 75-g OGTT, according to which any one of the following conditions confers the diagnosis of GDM: fasting (0-h) blood glucose of $\geq 92 \mathrm{mg} / \mathrm{dl}(5 \cdot 1 \mathrm{mmol} / \mathrm{l})$, 1-h post-load plasma glucose of $\geq 180 \mathrm{mg} / \mathrm{dl}(10 \cdot 0 \mathrm{mmol} / \mathrm{l})$ or 2 -h postload plasma glucose of $\geq 153 \mathrm{mg} / \mathrm{dl}(8.5 \mathrm{mmol} / \mathrm{l})^{(30)}$.

Clinical data such as pre-pregnancy body weight, weight gain to 24-28 weeks, previous history of GDM and pancreatic disease, and family history of diabetes were recorded for each pregnant woman. In all women, maternal weight and height were obtained by direct measurement on the examination day, while information on pre-pregnancy weight was self-reported by each woman. The gestational age was estimated from the last menstrual period, and was confirmed or corrected by ultrasonography.

All participants provided written informed consent and the study was approved by the Nanfang Hospital Ethics Committee.

\section{Physical activity}

The physical activity of all women was evaluated with the International Physical Activity Questionnaire (IPAQ) $)^{(31)}$. This questionnaire included four domains of physical activity: (i) occupational (seven items); (ii) transportation (six items); (iii) household/gardening (six items); and (iv) leisure-time activities (six items). The questionnaire also included two questions about the time spent sitting as an indicator of sedentary behaviour. The number of days per week and the time spent walking per day as well as moderate and vigorous activities from all four domains were recorded. Computation of the total score (expressed as metabolic equivalents of task (MET) per week) requires recording of duration (in minutes) and frequency (days per week) of walking, moderate-intensity and vigorous-intensity activities. The following values were used for analysis of the IPAQ data: walking at work $=3.3$ MET; cycling for transportation $=6.0 \mathrm{MET}$; moderate yard work $=4.0 \mathrm{MET}$; and vigorous intensity in leisure $=8.0$ MET. Using these values, scores were calculated and ranked as follows:

Walking at work (MET-min/week)

$$
\begin{gathered}
=3.3 \times \text { walking at work minutes } \\
\times \text { days per week }
\end{gathered}
$$

Cycling for transportation (MET-min/week)

$$
\begin{aligned}
=6.0 & \times \text { cycling for transportation minutes } \\
& \times \text { days per week }
\end{aligned}
$$

Moderate yard work (MET-min/week)

$$
\begin{aligned}
=4 \cdot 0 & \times \text { moderate yard work minutes } \\
& \times \text { days per week }
\end{aligned}
$$

Vigorous intensity in leisure (MET-min/week)

$$
\begin{gathered}
=8.0 \times \text { vigorous intensity inleisure minutes } \\
\times \text { days per week }
\end{gathered}
$$


The sum of all activities (walking at work, cycling for transportation, moderate yard work and vigorous intensity in leisure) represents the total weekly physical activity for each participant (MET-min/week scores) ${ }^{(31)}$. Based on these calculations, three categorical scores were arbitrarily defined: low ( $\leq 600 \mathrm{MET}-\mathrm{min} /$ week), middle (600-1500 MET-min/week) and high ( $\geq 1500 \mathrm{MET}-\mathrm{min} /$ week).

\section{Dietary babits}

Interviews were completed for all women using an FFQ to evaluate their dietary habits, in which the dietary status from the beginning of the second trimester of pregnancy (12th gestational week) to the time of OGTT (24th to 28th gestational week) was investigated. The FFQ comprises eighty-one food items and the individual food items are aggregated into thirteen food groups that cover $90 \%$ of foods commonly consumed in urban Guangzhou. In the fruit group, commonly consumed fruits were classified into three categories according to their glycaemic index (GI) value. High-GI fruits are defined as local tropical fruits such as litchi, longan, sugan cane, Hainan banana and durian, etc. Energy-dense snack foods (locally featured) in the dessert group included Cantonese Dim Sum such as lotus leaf glutinous rice, creamy egg yolk bun, egg custard tart and steamed lotus seed purée, baozi stuffed with red bean paste/creamy custard, etc. The FFQ has been previously tested for validity and reproducibility in similar populations signifying its appropriate use in this population $^{(32)}$. Each woman was asked to report her usual frequency of consumption as the number of times per day, per week, per month, per year, or never during the pregnancy and the average amount of food intake each time. Food intake data for each woman were then inputted into a computerized program (produced by Weizhida Electronic Technology Co., Xiangfan, Hubei Province, China) for calculating the amount of energy intake and dietary nutrient content (based on the tables of Chinese food composition ${ }^{(33)}$ ).

\section{Cognitive view on pregnancy bealth}

Using a structured questionnaire, face-to-face interviews were conducted by three trained interviewers to obtain information on maternal sociodemographic characteristics (age, residence, marital status, occupation and education). The questionnaire included questions about the pregnant woman's appetite in the first trimester, as well as her opinion about nausea and vomiting-related fetal health problems, dietary behaviour following the ending of morning sickness, and whether she received adequate prenatal care, both pregnancy- and diet-related health educations. Epdata3.02 (Military Medical Science Press, Beijing, China) was adopted to manage the data in the survey.

\section{Statistical analysis}

Descriptive characteristics of pregnant women were reported using means and standard deviations. One-way
ANOVA was performed to assess the univariate differences among continuous variables. The $\chi^{2}$ test was used to compare the observed frequencies between various groups. Multiple logistic regression analysis was used to examine the associations of dietary habits, physical activity and cognitive views on pregnancy health with GDM after adjusting for the factors that were significant in univariate analyses. Statistical comparisons were considered significant at $P<0 \cdot 05$. All analyses were performed using the statistical software package SPSS version $15 \cdot 0$.

\section{Results}

\section{Basic clinical characteristics of observed participants}

Of the 571 women, 539 (94\%) cases were fully interviewed and thus evaluated in the present study. The investigated women underwent an OGTT at 26.12 (SD 1.91) weeks' gestation, 470 ( $87 \%$ ) of them were identified as GDM negative $\left(\mathrm{GDM}^{-}\right)$and sixty-nine $(13 \%)$ of them as GDM positive $\left(\mathrm{GDM}^{+}\right)$. The basic clinical characteristics of the women and the diagnosis of GDM according to OGTT results are shown in Table 1. Compared with the $\mathrm{GDM}^{-}$women, $\mathrm{GDM}^{+}$women were older, heavier (before pregnancy) and had greater weight gain during pregnancy (all $P<0 \cdot 05$ ).

\section{Nutritional status of participants with and witbout gestational diabetes mellitus}

The dietary intakes of macronutrients and other energycarrying foods in women with and without GDM are shown in Table 2. No significant differences were observed between the $\mathrm{GDM}^{+}$and $\mathrm{GDM}^{-}$groups in the intake of energy, macronutrients, fibre or cholesterol. The amounts of low- and middle-GI fruits consumed daily in the two groups were not statistically different; however, significantly higher intake of high-GI fruits was observed in $\mathrm{GDM}^{+}$women than in $\mathrm{GDM}^{-}$women $(P=0 \cdot 010)$. With regard to dietary energy-dense snack foods, women in the $\mathrm{GDM}^{+}$group also demonstrated an increased daily intake compared with those in the $\mathrm{GDM}^{-}$group $(P=0 \cdot 002)$.

\section{Plasma glucose levels in participants according to physical activity and BMI}

The intensity of physical activity in $\mathrm{GDM}^{+}$women was statistically lower than that in $\mathrm{GDM}^{-}$women $(833.52$ (sD 384.64) v. 966.31 (SD 455.92) MET-min/week, respectively; $P=0 \cdot 020)$. We next investigated the levels of fasting $(0 \mathrm{~h}), 1-\mathrm{h}$ and 2 -h post-load plasma glucose in normal-weight, overweight and obese women with low, middle or high physical activity. The comparisons between different physical activity intensities at each body weight level showed no statistical difference in the fasting, 1-h or 2-h post-load plasma glucose values (Table 3). However, in 
Table 1 Basic clinical characteristics of participants according to GDM status: pregnant women, Guangzhou, Guangdong Province, China

\begin{tabular}{|c|c|c|c|c|c|c|}
\hline & \multicolumn{2}{|c|}{$\mathrm{GDM}^{+}(n$ 69) } & \multicolumn{2}{|c|}{$\mathrm{GDM}^{-}(n$ 470) } & \multirow[b]{2}{*}{$F$} & \multirow[b]{2}{*}{$P$} \\
\hline & Mean & SD & Mean & SD & & \\
\hline Age (years) & $29 \cdot 54$ & $4 \cdot 15$ & $28 \cdot 29$ & $4 \cdot 03$ & $5 \cdot 692$ & 0.017 \\
\hline Pre-pregnancy BMI (kg/m²) & $21 \cdot 25$ & $3 \cdot 05$ & $20 \cdot 03$ & $2 \cdot 36$ & $14 \cdot 689$ & 0.000 \\
\hline Weight gain during pregnancy $(\mathrm{kg})$ & $11 \cdot 13$ & $2 \cdot 57$ & $9 \cdot 71$ & $3 \cdot 15$ & $12 \cdot 639$ & 0.001 \\
\hline
\end{tabular}

$\mathrm{GDM}^{+/-}$, women with/without gestational diabetes mellitus according to results of an oral glucose tolerance test at the 24th to 28 th gestational week. The investigated women were on average $28 \cdot 53$ (SD 4.13) years of age, with pre-pregnancy BMI of $20 \cdot 24(\mathrm{SD} 2 \cdot 51) \mathrm{kg} / \mathrm{m}^{2}$ and weight gain of $9 \cdot 92(\mathrm{SD} 3 \cdot 14) \mathrm{kg}$ at 26 th gestational week.

Table 2 Nutrient and food intakes of participants according to GDM status: pregnant women, Guangzhou, Guangdong Province, China

\begin{tabular}{|c|c|c|c|c|c|c|}
\hline & \multicolumn{2}{|c|}{$\mathrm{GDM}^{+}(n 69)$} & \multicolumn{2}{|c|}{$\mathrm{GDM}^{-}(n$ 470) } & \multirow[b]{2}{*}{$F$} & \multirow[b]{2}{*}{$P$} \\
\hline & Mean & SD & Mean & SD & & \\
\hline Energy (kJ/d) & 10267 & 1962 & 10066 & 2382 & 0.445 & 0.505 \\
\hline Energy $(\mathrm{kcal} / \mathrm{d})$ & $2453 \cdot 90$ & 468.93 & $2405 \cdot 93$ & $569 \cdot 27$ & 0.445 & 0.505 \\
\hline Protein $(\mathrm{g} / \mathrm{d})$ & $80 \cdot 30$ & $22 \cdot 93$ & $79 \cdot 12$ & $25 \cdot 40$ & $0 \cdot 135$ & 0.714 \\
\hline$\%$ of energy & \multicolumn{2}{|c|}{$10 \cdot 65$} & \multicolumn{2}{|c|}{$10 \cdot 55$} & & \\
\hline $\begin{array}{l}\text { Fat }(\mathrm{g} / \mathrm{d}) \\
\% \text { of energy }\end{array}$ & \multicolumn{2}{|c|}{$\begin{array}{r}96 \cdot 91 \\
35.52\end{array}$} & \multicolumn{2}{|c|}{$35 \cdot 64$} & $0 \cdot 166$ & 0.684 \\
\hline Carbohydrate (g/d) & $329 \cdot 77$ & $77 \cdot 78$ & $323 \cdot 52$ & $83 \cdot 44$ & 0.343 & 0.558 \\
\hline$\%$ of energy & \multicolumn{2}{|c|}{53.83} & \multicolumn{2}{|c|}{$53 \cdot 81$} & & \\
\hline SFA (\%) & $4 \cdot 41$ & $1 \cdot 76$ & $5 \cdot 27$ & $1 \cdot 40$ & $0 \cdot 153$ & 0.696 \\
\hline MUFA (\%) & $7 \cdot 28$ & $2 \cdot 44$ & $7 \cdot 34$ & $2 \cdot 10$ & 0.048 & 0.826 \\
\hline PUFA (\%) & $9 \cdot 71$ & $4 \cdot 62$ & $9 \cdot 23$ & $4 \cdot 17$ & 0.775 & 0.379 \\
\hline Fibre $(\mathrm{g} / \mathrm{d})$ & $17 \cdot 08$ & $4 \cdot 10$ & $18 \cdot 60$ & $5 \cdot 75$ & $4 \cdot 474$ & 0.055 \\
\hline Cholesterol $(\mathrm{g} / \mathrm{d})$ & $488 \cdot 48$ & $227 \cdot 91$ & $477 \cdot 80$ & $231 \cdot 32$ & $0 \cdot 129$ & 0.720 \\
\hline Low-GI fruits (g/d) & $362 \cdot 42$ & $176 \cdot 81$ & $352 \cdot 53$ & $165 \cdot 68$ & $0 \cdot 152$ & 0.643 \\
\hline Middle-Gl fruits $(\mathrm{g} / \mathrm{d})$ & $60 \cdot 72$ & 86.99 & $56 \cdot 19$ & $79 \cdot 40$ & $0 \cdot 191$ & 0.662 \\
\hline High-Gl fruits ( $\mathrm{g} / \mathrm{d})$ & $85 \cdot 52$ & $200 \cdot 33$ & 43.90 & $109 \cdot 95$ & $6 \cdot 656$ & 0.010 \\
\hline Energy-dense snack foods $(\mathrm{g} / \mathrm{d})$ & $41 \cdot 43$ & $5 \cdot 76$ & $32 \cdot 48$ & $13 \cdot 13$ & $34 \cdot 143$ & 0.002 \\
\hline Nuts $(g / d)$ & $31 \cdot 80$ & $33 \cdot 02$ & $29 \cdot 80$ & $32 \cdot 62$ & $0 \cdot 224$ & 0.636 \\
\hline Oils $(g / d)$ & $28 \cdot 46$ & $9 \cdot 34$ & $28 \cdot 63$ & $9 \cdot 44$ & 0.020 & 0.888 \\
\hline
\end{tabular}

$\mathrm{GDM}^{+/-}$, women with/without gestational diabetes mellitus according to results of an oral glucose tolerance test at the 24th to 28th gestational week; Gl, glycaemic index.

The data in percentage indicate the proportional energy contribution of protein, fat or carbohydrate. The mean daily energy intake of the whole cohort was 10092 (SD 2332) kJ/d (2412.12 (SD 557.34) kcal), with carbohydrate, fat and protein taking the proportion of $53 \cdot 81 \%$, $35 \cdot 63 \%$ and $10 \cdot 56 \%$, respectively.

the women with low physical activity, the frequency of GDM diagnosed according to 1-h post-load plasma glucose in the overweight group was higher than in the normal-weight group $(12 \cdot 74 \% v .87 \cdot 26 \%, P=0 \cdot 034)$.

\section{Comparison of cognitive views on pregnancy bealth between participants with and witbout gestational diabetes mellitus}

The cognitive views on pregnancy health between $\mathrm{GDM}^{+}$ and $\mathrm{GDM}^{-}$women are compared in Table 4 . Women who regarded nausea and vomiting-caused insufficient food intake as relevant to fetal health problems were associated with an increased risk for GDM $(P=0 \cdot 018)$. Moreover, women who increased their dietary intake after morning sickness were at a higher risk of GDM $(P=0 \cdot 038)$. On the other hand, $\mathrm{GDM}^{-}$women reported a higher frequency of receipt of pregnancy- and dietrelated health education than $\mathrm{GDM}^{+}$women $(P=0 \cdot 043)$. Compared with the $\mathrm{GDM}^{-}$group, the $\mathrm{GDM}^{+}$group was more likely to report having a family history of diabetes $(P=0 \cdot 014)$. Nevertheless, the frequencies of family history with pancreatic disease between the two groups did not have statistical difference $(P=0 \cdot 286)$.

\section{Multiple logistic regression analysis of associations of dietary habits, physical activity and cognitive views on pregnancy bealth with gestational diabetes mellitus}

The adjusted odds ratios for factors that were significantly associated with GDM risk are summarized in Table 5. Women with higher BMI before pregnancy $(\mathrm{OR}=1 \cdot 151$; $95 \%$ CI $1 \cdot 035,1 \cdot 280, P=0 \cdot 009)$ and with a greater weight gain during pregnancy $(\mathrm{OR}=1 \cdot 096$; $95 \%$ CI 1·004, 1·280, $P=0 \cdot 041)$ were at an increased risk of GDM. Compared with women without a family history of diabetes, those with a positive family history were at increased risk of GDM $\quad(\mathrm{OR}=2 \cdot 101 ; 95 \%$ CI $1 \cdot 040,4 \cdot 244, \quad P=0 \cdot 038)$. Increased consumption of specific foods such as energydense snack foods and high-GI fruits was associated with increased risk of GDM $(\mathrm{OR}=1 \cdot 050 ; 95 \%$ CI 1.029, 1.071, $P=0.001$ and $\mathrm{OR}=1 \cdot 002 ; 95 \%$ CI $1 \cdot 000,1 \cdot 004, P=0 \cdot 017$, respectively). In addition, women who believed that nausea 
Table 3 Plasma glucose levels in participants according to physical activity level and BMI: pregnant women, Guangzhou, Guangdong Province, China

\begin{tabular}{|c|c|c|c|c|c|c|c|c|}
\hline & \multicolumn{2}{|c|}{ Low physical activity } & \multicolumn{2}{|c|}{ Middle physical activity } & \multicolumn{2}{|c|}{ High physical activity } & \multirow[b]{2}{*}{$F$} & \multirow[b]{2}{*}{$P$} \\
\hline & Mean & SD & Mean & SD & Mean & SD & & \\
\hline \multicolumn{9}{|c|}{ 0-h (fasting) PG (mmol/l) } \\
\hline Normal weight & $4 \cdot 31$ & 0.53 & $4 \cdot 31$ & 0.45 & $4 \cdot 25$ & $0 \cdot 40$ & $2 \cdot 066$ & 0.128 \\
\hline Overweight & $4 \cdot 38$ & $0 \cdot 75$ & $4 \cdot 39$ & $0 \cdot 40$ & $4 \cdot 41$ & $0 \cdot 34$ & 0.009 & 0.991 \\
\hline Obese & 3.97 & 0.29 & $4 \cdot 65$ & $0 \cdot 78$ & - & - & $2 \cdot 178$ & 0.236 \\
\hline \multicolumn{9}{|c|}{ 1-h post-load PG (mmol/l) } \\
\hline Normal weight & $7 \cdot 18$ & $1 \cdot 70$ & $7 \cdot 07$ & $1 \cdot 60$ & $6 \cdot 98$ & $1 \cdot 71$ & $0 \cdot 260$ & $0 \cdot 771$ \\
\hline Overweight & $8 \cdot 66$ & $2 \cdot 17$ & 8.00 & $1 \cdot 68$ & - & - & 0.458 & 0.641 \\
\hline Obese & $8 \cdot 30$ & $1 \cdot 14$ & $7 \cdot 83$ & $1 \cdot 25$ & $7 \cdot 96$ & $1 \cdot 41$ & $2 \cdot 461$ & $0 \cdot 125$ \\
\hline \multicolumn{9}{|c|}{ 2-h post-load PG (mmol/l) } \\
\hline Normal weight & $6 \cdot 59$ & 1.53 & $6 \cdot 62$ & $1 \cdot 20$ & $6 \cdot 41$ & 1.07 & 0.671 & 0.512 \\
\hline Overweight & $7 \cdot 46$ & $1 \cdot 23$ & $6 \cdot 89$ & $1 \cdot 23$ & $7 \cdot 05$ & $1 \cdot 25$ & 0.592 & 0.558 \\
\hline Obese & $7 \cdot 30$ & $1 \cdot 70$ & $6 \cdot 67$ & $1 \cdot 52$ & - & - & $0 \cdot 193$ & 0.690 \\
\hline
\end{tabular}

PG, plasma glucose.

Normal weight, pre-pregnancy $\mathrm{BMI}=18 \cdot 5-23.9 \mathrm{~kg} / \mathrm{m}^{2}$; overweight, pre-pregnancy $\mathrm{BMI}=24 \cdot 0-27 \cdot 9 \mathrm{~kg} / \mathrm{m}^{2} ;$ obese, pre-pregnancy $\mathrm{BMI} \geq 28 \cdot 0 \mathrm{~kg} / \mathrm{m}^{2}$ (the specific BMI reference values for Chinese as defined by the China Public Union of Nutrition).

Table 4 Comparison of cognitive views on pregnancy health of participants according to GDM status: pregnant women, Guangzhou, Guangdong Province, China

\begin{tabular}{|c|c|c|c|c|c|c|c|c|c|c|}
\hline \multirow[b]{3}{*}{ Question in interview on pregnant women } & \multicolumn{4}{|c|}{$\mathrm{GDM}^{+}(n$ 69) } & \multicolumn{4}{|c|}{$\mathrm{GDM}^{-}(n 470)$} & \multirow[b]{3}{*}{$\chi^{2}$} & \multirow[b]{3}{*}{$P$} \\
\hline & \multicolumn{2}{|c|}{ Yes } & \multicolumn{2}{|c|}{ No } & \multicolumn{2}{|c|}{ Yes } & \multicolumn{2}{|c|}{ No } & & \\
\hline & $n$ & $\%$ & $n$ & $\%$ & $n$ & $\%$ & $n$ & $\%$ & & \\
\hline Previous history of pancreatic disease & 1 & $1 \cdot 44$ & 68 & $98 \cdot 56$ & 2 & 0.01 & 468 & 99.99 & $1 \cdot 139$ & $0 \cdot 286$ \\
\hline Family history of diabetes & 16 & $23 \cdot 19$ & 53 & $76 \cdot 81$ & 58 & $12 \cdot 34$ & 412 & $87 \cdot 66$ & 5.978 & 0.014 \\
\hline Probability of morning sickness causing fetal health problems & 44 & $63 \cdot 77$ & 25 & $36 \cdot 23$ & 228 & 48.51 & 242 & $51 \cdot 49$ & 5.603 & 0.018 \\
\hline Unlimited food intake after the ending of morning sickness & 44 & $63 \cdot 77$ & 25 & $36 \cdot 23$ & 237 & $50 \cdot 42$ & 233 & $49 \cdot 58$ & $4 \cdot 292$ & 0.038 \\
\hline Receipt of health education about pregnancy and diet & 38 & $55 \cdot 07$ & 31 & 44.93 & 317 & $67 \cdot 45$ & 153 & $32 \cdot 55$ & $4 \cdot 098$ & 0.043 \\
\hline
\end{tabular}

$\mathrm{GDM}^{+/-}$, women with/without gestational diabetes mellitus according to results of an oral glucose tolerance test at the 24th to 28 th gestational week.

The $P$ values were calculated by comparison between $\mathrm{GDM}^{+}$and $\mathrm{GDM}^{-}$women using the $\chi^{2}$ test.

Table 5 Multiple logistic regression analysis of the associations of dietary habits, physical activity and cognitive views on pregnancy health with gestational diabetes mellitus: pregnant women, Guangzhou, Guangdong Province, China

\begin{tabular}{|c|c|c|c|c|c|c|}
\hline Characteristic & $B$ & SE & Wald & $P$ & OR & $95 \% \mathrm{Cl}$ \\
\hline Age (years) & 0.047 & 0.035 & $1 \cdot 844$ & $0 \cdot 175$ & $1 \cdot 048$ & $1 \cdot 000,1 \cdot 120$ \\
\hline Pre-pregnancy BMI (kg/m²) & $0 \cdot 141$ & 0.054 & $6 \cdot 749$ & 0.009 & $1 \cdot 151$ & $1 \cdot 035,1 \cdot 280$ \\
\hline Weight gain during pregnancy $(\mathrm{kg})$ & 0.092 & 0.045 & $4 \cdot 157$ & 0.041 & $1 \cdot 096$ & $1 \cdot 004,1 \cdot 280$ \\
\hline Positive family history of diabetes & 0.742 & 0.359 & $4 \cdot 286$ & 0.038 & $2 \cdot 101$ & $1 \cdot 040,4 \cdot 244$ \\
\hline High-Gl fruits $(g / d)$ & 0.002 & 0.001 & $5 \cdot 719$ & 0.017 & $1 \cdot 002$ & $1 \cdot 000,1 \cdot 004$ \\
\hline Energy-dense snack foods (g/d) & 0.049 & $0 \cdot 010$ & $22 \cdot 304$ & 0.001 & $1 \cdot 050$ & $1 \cdot 029,1 \cdot 071$ \\
\hline Physical activity (MET-min/week) & -0.001 & 0.000 & $2 \cdot 339$ & $0 \cdot 126$ & 0.999 & $0.990,1.000$ \\
\hline Probability of morning sickness causing fetal health problems & 0.648 & 0.302 & $4 \cdot 604$ & 0.032 & 1.911 & $1 \cdot 058,3 \cdot 453$ \\
\hline Unlimited food intake after the ending of morning sickness & 0.388 & $0 \cdot 302$ & $0 \cdot 302$ & $0 \cdot 199$ & $1 \cdot 474$ & $0 \cdot 815,2 \cdot 667$ \\
\hline Receipt of health education about pregnancy and diet & -0.455 & $0 \cdot 293$ & $2 \cdot 408$ & $0 \cdot 121$ & 0.634 & $0 \cdot 357,1 \cdot 127$ \\
\hline
\end{tabular}

GI, glycaemic index; MET, metabolic equivalents of task.

and vomiting-caused reduction in food intake would cause fetal health problems were associated with an increased risk of GDM (OR $=1 \cdot 911 ; 95 \%$ CI 1·058, 3.453, $P=0 \cdot 032)$.

\section{Discussion}

In accordance with previous reports, the present study observed that some factors, such as increased pre-pregnancy
BMI, greater weight gain during pregnancy and family history of diabetes, were strongly independent predictors of reduced glucose tolerance. In the present study we further observed that higher intakes of high-GI fruit and energy-dense snack foods during pregnancy elevated GDM risk. It was also found that increased physical activity was associated with a reduction of 1-h post-load plasma glucose in pregnant women. Lastly, unlimited food intake starting from the second trimester, due to an 
incorrect belief that morning sickness-caused reduction in food intake in the first trimester would cause fetal health problems, demonstrated a correlation with increased risk of GDM. Overall, the results of our study point to the significance of health education regarding the control of food intake during pregnancy, especially after the first trimester, and correct cognitive views on pregnancyrelated health care.

It appears contradictory that our finding of increased intakes of high-GI fruits and energy-dense snack foods as contributory to GDM were not paralleled with an increased intake of total energy. In fact, both energy-dense snack foods (dietary fibre-free and usually supplemented with substantial amounts of sucrose, a disaccharide that easily hydrolyses to glucose in the gastrointestinal tract) and high-GI fruits are of high GI levels. While it is commonly accepted that high-GI foods are more rapidly digested and absorbed in the gastrointestinal tract and thus are more contributory to diabetes than regular foods, our study only measured the plasma glucose level at a single point in time and investigated dietary status from the beginning of the second trimester of pregnancy (12th gestational week) to the time of the OGTT (24th to 28th gestational week, covering only about a third of the gestation period); additionally, the pre-pregnancy energy consumption and dietary habits were not obtained. Therefore, the exact risk factors responsible for GDM may be complicated, wherein cognitive views and increased intake of high-GI foods may take a part; however, whether the increase in high-GI foods without the accompanying increase in total energy could be contributory to GDM remains to be investigated, probably including the potential involvement of prepregnancy dietary status as well. Additionally, the present study did not identify the risk factors for greater weight gain in women with GDM exactly, namely increased energy intake, decreased physical activity, or both of them, and this may be investigated in future studies.

In China, due to the implementation of the 'one-child policy' and the government's encouragement to females for a later marital age ( $>23$ years) and later childbearing ( $>24$ years), pregnant woman in a family, especially at an advanced age, are regarded as the most important person to be looked after by other family members. Most Chinese pregnant women stop or reduce the amount of housework, some even quit their jobs as soon as they find out they are pregnant ${ }^{(34,35)}$, and as a result their sedentary activities increase ${ }^{(35)}$. Even if they are used to doing housework, such as cooking, grocery shopping, washing the dishes and sweeping the floor before getting pregnant, it becomes their family members' tasks thereafter. Moreover, according to Chinese traditional taboos, intensive limb activities, such as mopping the floor, moving furniture and carrying wet clothes (for drying them in the sun at open sites), may cause miscarriage or premature birth. Therefore, pregnant women are usually prevented from doing these things by their mother-in-law even if they would like to do them according to the modern education they have received ${ }^{(35)}$. These sedentary lifestyles may be associated with an increased risk of weight gain, obesity, GDM and pregnancy-induced hyperten$\operatorname{sion}^{(36,37)}$. In fact, a much larger pregnant population may have abnormal glucose tolerance than the proportion with GDM, which is also associated with maternal complications such as pregnant hypotension, pre-eclampsia, macrosomia and birth trauma ${ }^{(11,27)}$. Therefore most pregnant Chinese women should have the opportunity to be educated and understand they do not have to quit regular daily physical activity, including housework; on the contrary, for some who are engaged in sedentary job categories, for example an office clerk, it is necessary to add (but not avoid) some physical exercise.

The native people investigated in the present study are accustomed to consuming Cantonese Dim Sum and tropical fruits which are energy-dense and/or high-GI foods ${ }^{(38)}$. According to our results, high-GI and energydense foods, as the common snacks in all participants between regular meals, were more consumed by $\mathrm{GDM}^{+}$ women. Excessive intake of these foods has been proposed to increase the body's demand for insulin, and this may lead to insulin resistance, impaired pancreatic $\beta$-cell function and eventually type 2 diabetes, as specialized by $\mathrm{GDM}^{(39,40)}$.

Since, as described above, incorrect cognitive views on pregnancy-related health care may be associated with GDM in Chinese pregnant women, for the prevention and control of GDM, it is important that individualized health education, dietary and exercise advice is offered to pregnant as well as pre-pregnant women. Due to the limited time for each pregnant woman in out-patient services in China, it is highly recommended to establish a GDM education group in each hospital. This group should include dietitians, maternity nurses and obstetricians, for regular consultation services, particularly for correction of the wrong points of view on pregnancyrelated adjustments in diet and physical activities. This health education is not only a task for hospitals, but also community health carers. In summary, an intensive health education may help pregnant women, especially those with GDM, to understand their health conditions and may guide them in choosing a healthy (case-specific) lifestyle with a low risk of GDM.

\section{Study limitations}

Our study has some limitations. First, the relatively small number of observed cases of GDM limited our ability to reach more solid conclusions. Second, as the results of our assessment on physical activity and diet are specific to only a small window of a much longer period of gestation, our study cannot reflect situations in other gestational stages than 24 to 28 weeks. Moreover, as in any investigative study on human populations which relies on self-reported questions, the obtained dietary 
status and the intensity of physical activities were also self-reported and with relatively low accuracy compared with experimentally controlled studies.

\section{Conclusion}

Intensive health education that provides guidance on reasonable dietary behaviour and adequate physical activities is highly in demand for the control of GDM, and further work is needed to better understand the relationship between diet, physical activity, health education and glucose disturbances during pregnancy.

\section{Acknowledgements}

Sources of funding: This research was supported by the President Foundation of Nanfang Hospital, Southern Medical University (NO: 2010C016). The funder had no role in the design, analysis or writing of this article. Conflicts of interests: The authors declare that they have no competing interests. Authors' contributions: Q.L. and R.X. have contributed equally to this work. Q.L., Y.L., L.S. and B.L. contributed to the design of the research. Q.L., R.X. and J.C. performed the interviews of participants with questionnaires. L.W. performed the statistical analysis. Q.L. and R.X. drafted the manuscript. Q.L. was supervised by Y.L. and B.L. in the study idea and survey; B.L. also supervised the research and statistical analysis. Y.L. revised the manuscript. All authors read and approved the final manuscript. Acknowledgements: The authors thank all participants in the study. The authors greatly appreciate support from the Department of Gynecology and Obstetrics and the Department of Endocrinology at Nanfang Hospital.

\section{References}

1. American Diabetes Association (2004) Gestational diabetes mellitus. Diabetes Care 27, Suppl. 1, S88-S90.

2. Dabelea D, Snell-Bergeon JK, Hartsfield CL et al. (2005) Increasing prevalence of gestational diabetes mellitus (GDM) over time and by birth cohort: Kaiser Permanente of Colorado GDM Screening Program. Diabetes Care 28, 579-584.

3. Kjos SL, Buchanan TA, Greenspoon JS et al. (1990) Gestational diabetes mellitus: the prevalence of glucose intolerance and diabetes mellitus in the first two months post partum. Am J Obstet Gynecol 163, 93-98.

4. Kwik M, Seeho SK, Smith C et al. (2007) Outcomes of pregnancies affected by impaired glucose tolerance. Diabetes Res Clin Pract 77, 263-268.

5. World Diabetes Foundation, Global Alliance for Women's Health (2009) Diabetes, Women, and Development: meeting summary, expert recommendations for policy action, conclusions, and follow-up actions. Int J Gynaecol Obstet 104, Suppl. 1, S46-S50.

6. Jiwani A, Marseille E, Lohse N et al. (2012) Gestational diabetes mellitus: results from a survey of country prevalence and practices. J Matern Fetal Neonatal Med 25, 600-610.
7. Veeraswamy S, Vijayam B, Gupta VK et al. (2012) Gestational diabetes: the public health relevance and approach. Diabetes Res Clin Pract 97, 350-358.

8. Metzger BE, Lowe LP, Dyer AR et al. (2008) Hyperglycemia and adverse pregnancy outcomes. $N$ Engl J Med 358, 1991-2002.

9. Sermer M, Naylor CD, Gare DJ et al. (1995) Impact of increasing carbohydrate intolerance on maternal-fetal outcomes in 3637 women without gestational diabetes. The Toronto Tri-Hospital Gestational Diabetes Project. Am J Obstet Gynecol 173, 146-156.

10. Tallarigo L, Giampietro O, Penno G et al. (1986) Relation of glucose tolerance to complications of pregnancy in nondiabetic women. $N$ Engl J Med 315, 989-992.

11. Scholl TO, Sowers M, Chen X et al. (2001) Maternal glucose concentration influences fetal growth, gestation, and pregnancy complications. Am J Epidemiol 154, 514-520.

12. Vohr BR, McGarvey ST \& Tucker R (1999) Effects of maternal gestational diabetes on offspring adiposity at 4-7 years of age. Diabetes Care 22, 1284-1291.

13. Ma RC \& Chan JC (2009) Pregnancy and diabetes scenario around the world: China. Int J Gynaecol Obstet 104, Suppl. 1, S42-S45.

14. Yang X, Hsu-Hage B, Zhang $\mathrm{H}$ et al. (2002) Gestational diabetes mellitus in women of single gravidity in Tianjin City, China. Diabetes Care 25, 847-851.

15. Zhang F, Dong L, Zhang CP et al. (2011) Increasing prevalence of gestational diabetes mellitus in Chinese women from 1999 to 2008. Diabet Med 28, 652-657.

16. Koren G, Maltepe C \& Gow R (2011) Therapeutic choices for nausea and vomiting of pregnancy: a critical review of a systematic review. J Obstet Gynaecol Can 33, 733-735.

17. Quinla JD \& Hill DA (2003) Nausea and vomiting of pregnancy. Am Fam Physician 68, 121-128.

18. Allen LH (2005) Multiple micronutrients in pregnancy and lactation: an overview. Am J Clin Nutr 81, issue 5, 1206S-1212S.

19. Latva-Pukkila U, Isolauri E \& Laitinen K (2010) Dietary and clinical impacts of nausea and vomiting during pregnancy. J Hum Nutr Diet 23, 69-77.

20. Wang Y, Mi J, Shan XY et al. (2007) Is China facing an obesity epidemic and the consequences? The trends in obesity and chronic disease in China. Int J Obes (Lond) 31, 177-188.

21. Yang W, Lu J, Weng J et al. (2010) Prevalence of diabetes among men and women in China. N Engl J Med 362, 1090-1101.

22. Kaiser L \& Allen LH (2008) Position of the American Dietetic Association: nutrition and lifestyle for a healthy pregnancy outcome. J Am Diet Assoc 108, 553-561.

23. Rudra CB, Williams MA, Lee IM et al. (2005) Perceived exertion during prepregnancy physical activity and preeclampsia risk. Med Sci Sports Exerc 37, 1836-1841.

24. Zhang C, Solomon CG, Manson JE et al. (2006) A prospective study of pregravid physical activity and sedentary behaviors in relation to the risk for gestational diabetes mellitus. Arch Intern Med 166, 543-548.

25. Bertolotto A, Volpe L, Calianno A et al. (2010) Physical activity and dietary habits during pregnancy: effects on glucose tolerance. J Matern Fetal Neonatal Med 23, 1310-1314.

26. Guelinckx I, Devlieger R, Mullie P et al. (2010) Effect of lifestyle intervention on dietary habits, physical activity, and gestational weight gain in obese pregnant women: a randomized controlled trial. Am J Clin Nutr 91, 373-380.

27. Oken E, Ning Y, Rifas-Shiman SL et al. (2006) Associations of physical activity and inactivity before and during pregnancy with glucose tolerance. Obstet Gynecol 108, 1200-1207.

28. Radesky JS, Oken E, Rifas-Shiman SL et al. (2008) Diet during early pregnancy and development of gestational diabetes. Paediatr Perinat Epidemiol 22, 47-59. 
29. Cheng HM, Pei JH \& Han F (2007) To investigate the effect of older pregnancy on the pregnant women and infants. J Pract Med 23, 1714-1715.

30. American Diabetes Association (2012) Diagnosis and classification of diabetes mellitus. Diabetes Care $\mathbf{3 5}$, Suppl. 1, S64-S71.

31. IPAQ Group (2005) Guidelines for Data Processing and Analysis of the International Physical Activity Questionnaire - Short and Long Forms. http://www.ipaq.ki.se/ scoring.pdf (accessed January 2010).

32. Zhang CX \& Ho SC (2009) Validity and reproducibility of a food frequency questionnaire among Chinese women in Guangdong province. Asia Pac J Clin Nutr 18, 240-250.

33. Yang YX, Wang GY \& Pan XC (2009) China Food Composition, pp. 1-193. Beijing: Beijing University Medical Press.

34. Ma G \& Kong L (2006) China Nutrition and Health Survey Report, pp. 130-218. Guangzhou: People's Medical Publishing House Press.
35. Su W, Li M, Yang H et al. (2009) Physical activities among pregnant women with normal glucose metabolism. J Nurs Sci 24, 9-12.

36. Nascimento SL, Surita FG \& Cecatti JG (2012) Physical exercise during pregnancy: a systematic review. Curr Opin Obstet Gynecol 24, 387-394.

37. Rooney BL, Schauberger CW \& Mathiason MA (2005) Impact of perinatal weight change on long-term obesity and obesity-related illnesses. Obstet Gynecol 106, 1349-1356.

38. Huang M (1996) Current situation and analysis of Guangzhou residents in 1990s. J Hyg Res 25, 378-379.

39. Salmeron J, Manson JE, Stampfer MJ et al. (1997) Dietary fiber, glycemic load, and risk of non-insulin-dependent diabetes mellitus in women. JAMA 277, 472-477.

40. Schulze MB, Liu S, Rimm EB et al. (2004) Glycemic index, glycemic load, and dietary fiber intake and incidence of type 2 diabetes in younger and middle-aged women. $A m J$ Clin Nutr 80, 348-356. 